\title{
Polar cap patch segmentation of the tongue of ionization in the morning convection cell
}

\author{
Q.-H. Zhang, ${ }^{1,2}$ B.-C. Zhang, ${ }^{1}$ J. Moen, ${ }^{3}$ M. Lockwood, ${ }^{4}$ I. W. McCrea, ${ }^{5}$ H.-G. Yang, ${ }^{1}$ \\ H.-Q. Hu, ${ }^{1}$ R.-Y. Liu, ${ }^{1}$ S.-R. Zhang, ${ }^{6}$ and M. Lester ${ }^{7}$ \\ Received 23 April 2013; revised 31 May 2013; accepted 31 May 2013; published 20 June 2013.
}

[1] Two types of poleward moving plasma concentration enhancements (PMPCEs) were observed during a sequence of pulsed reconnection events, both in the morning convection cell: Type L (low density) was associated with a cusp flow channel and seems likely to have been produced by ionization associated with particle precipitation, while Type $\mathrm{H}$ (high density) appeared to originate from the segmentation of the tongue of ionization by the processes which produced the Type $\mathrm{L}$ events. As a result, the Type $\mathrm{L}$ and Type H PMPCEs were interspersed, producing a complex density structure which underlines the importance of cusp flow channels as a mechanism for segmenting and structuring electron density in the cusp and shows the necessity of differentiating between at least two classes of electron density patches. Citation: Zhang, Q.-H., B.-C. Zhang, J. Moen, M. Lockwood, I. W. McCrea, H.-G. Yang, H.-Q. Hu, R.-Y. Liu, S.-R. Zhang, and M. Lester (2013), Polar cap patch segmentation of the tongue of ionization in the morning convection cell, Geophys. Res. Lett., 40, 2918-2922, doi:10.1002/grl.50616.

\section{Introduction}

[2] Polar cap patches are defined as islands of high density ionospheric plasma poleward of the auroral oval, surrounded by plasma of half the density or less [Crowley, 1996]. It was recognized at an early stage that midlatitude ionospheric plasma, produced by solar EUV, provided a viable reservoir of source plasma for both discrete polar cap patches or for a continuous tongue of ionization (TOI), with the plasma being drawn by convection into the polar cap [Knudsen, 1974; Foster et al., 2005]. Various mechanisms connected with the segmentation of the TOI have been proposed, but a difficulty for these patch segmentation mechanisms is to explain the low-density regions between patches [Lockwood et al., 2005]. One mechanism, considered to be dominant for patch

\footnotetext{
${ }^{1}$ SOA Key Laboratory for Polar Science, Polar Research Institute of China, Shanghai, China.

${ }^{2}$ Sate Key Laboratory of Space Weather, Chinese Academy of Sciences, Beijing, China.

${ }^{3}$ Department of Physics, University of Oslo, Oslo, Norway.

${ }^{4}$ Department of Meteorology, University of Reading, Reading, UK.

${ }^{5}$ SSTD, Rutherford-Appleton Laboratory, Didcot, UK.

${ }^{6}$ Haystack Observatory, Massachusetts Institute of Technology, Westford, Massachusetts, USA.

${ }^{7}$ Department of Physics and Astronomy, University of Leicester, Leicester, UK.

Corresponding author: Q.-H. Zhang, Polar Research Institute of China, No. 451, Jinqiao Rd., Pudong, Shanghai 200136, China. (zhangqinghe@pric. gov.cn)

(C)2013. American Geophysical Union. All Rights Reserved. 0094-8276/13/10.1002/grl.50616
}

segmentation, at least in the European sector [Carlson, 2012], involves a transient equatorward excursion of the open-closed boundary, entraining high-density solar EUV ionized plasma into the polar cap [Lockwood and Carlson, 1992; Lockwood et al., 2005; Zhang et al., 2011]. Ionization due to auroral particle precipitation has also been suggested as a source of polar cap patches [Weber et al., 1984]. MacDougall and Jayachandran [2007] suggested that auroral precipitation actually dominates polar cap patch production. The current literature is hence somewhat confusing, in that there is no standard nomenclature to differentiate "patches" caused by precipitation from those caused by the transport and segmentation of EUV-produced plasma. In this paper, we therefore prefer to use the terminology introduced by Zhang et al. [2011] and refer to all "patches" as poleward moving plasma concentration enhancements (PMPCEs) until they are classified. In the discussion section, we will illustrate the roles of both Type L PMPCEs (produced by precipitation) and Type H PMPCEs (produced by segmentation) in creating the complex density structures seen in the cusp region.

\section{Observations}

[3] Figures $1 \mathrm{a}$ and $1 \mathrm{~b}$ present an overview of interplanetary magnetic field (IMF) conditions measured by the ACE satellite during the period $0700-0830$ UT on 13 March 2004. The IMF data have all been lagged by $44.7 \mathrm{~min}$, which is the estimated propagation delay to Double Star TC-1 [Liu et al., 2005] which was located in the magnetosheath near the magnetopause at about 10 magnetic local time (MLT; cf. comparison of clock angles in Figure 1b). Before about $0746 \mathrm{UT}$, the IMF $B_{Z}$ component varied between -1 and $-6 \mathrm{nT}$ and the IMF $B_{Y}$ varied between 1 and $-2 \mathrm{nT}$ (see Figure 1a). At 0746 UT, $B_{Y}$ turned to steady negative around $-4 \mathrm{nT}$ and IMF $B_{Z}$ weakened (see Figure 1a).

[4] On 13 March 2004, the European Incoherent Scatter (EISCAT) Svalbard Radar (ESR) $32 \mathrm{~m}$ dish was pointing nearly toward geomagnetic north (azimuth $336^{\circ}$ ), at low elevation $\left(30^{\circ}\right)$. The beam of the EISCAT VHF radar, was also directed northward with a geographic azimuth of $359.45^{\circ}$ and an elevation of $30^{\circ}$. The combination of these radars allowed us to monitor the transportation of plasma from the subauroral region into the polar cap. Figures $1 \mathrm{c}-1 \mathrm{f}$ present data from the ESR and Figures $1 \mathrm{~h}-1 \mathrm{k}$ from the VHF radar for 0700-0830 UT ( $\sim 0930$ to 1100 MLT). ESR covered the latitude range $76.3^{\circ}$ and $82.5^{\circ}$ magnetic latitude (MLAT) with an increasing altitude from about 80 to $520 \mathrm{~km}$. In Figure 1c, a series of five PMPCEs moving along the beam to higher latitudes are highlighted by the solid black lines and marked by numbers 1-5. Although the plasma flow 


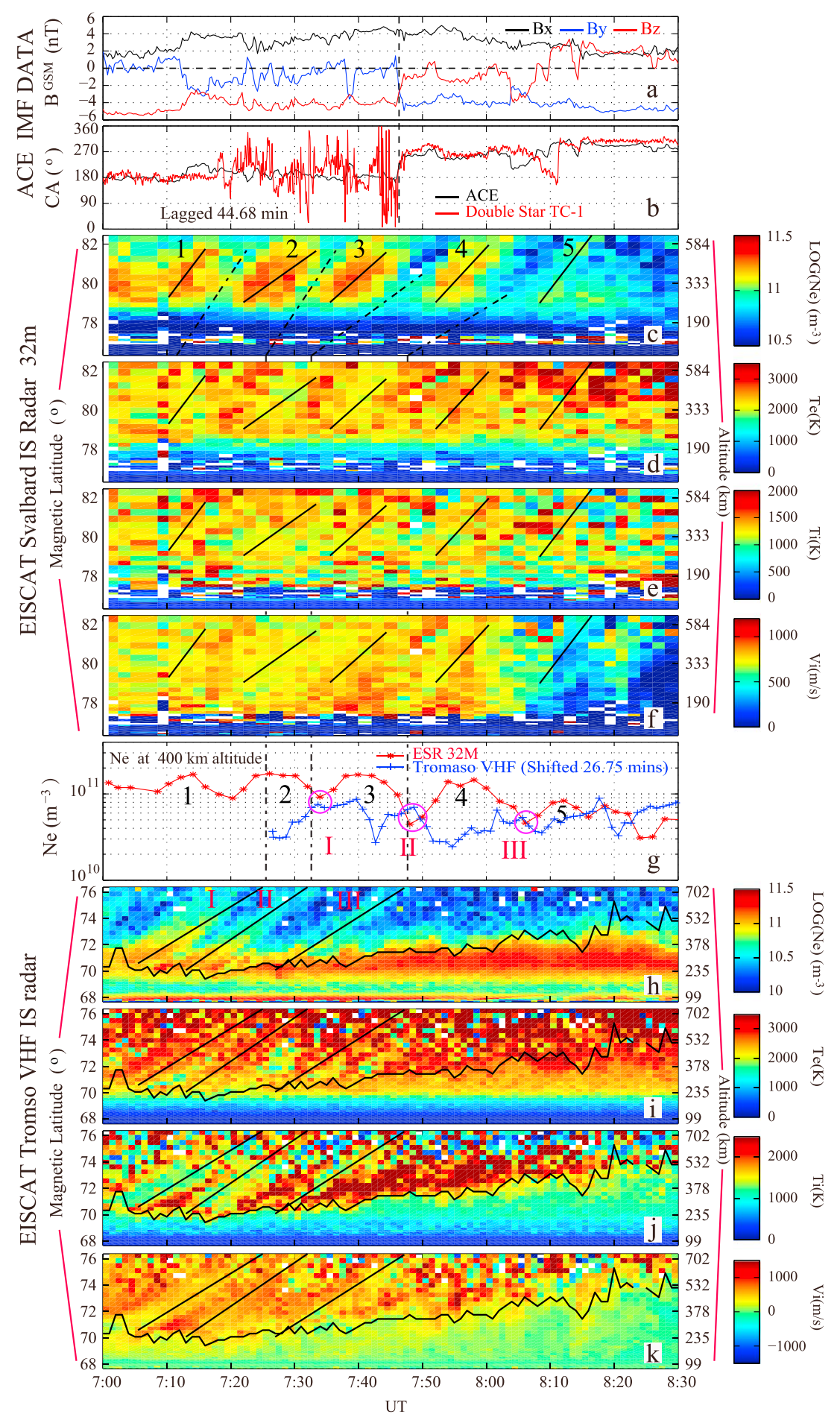

Figure 1. An overview of IMF conditions measured by the ACE satellite and plasma parameters observed by the northwarddirected ESR dish and the beam of the EISCAT VHF radar. From top to bottom: (a) the GSM IMF components; (b) the IMF clock angle, CA, together with the magnetosheath magnetic field clock angle from Double Star TC-1; (c, h) Ne, electron density; (d, i) Te, electron temperature; (e, j) Ti, ion temperature; and (f, $k$ ) line of sight velocity, Vi (positive away from the radar) as a function of time and magnetic latitude, together with $(\mathrm{g})$ the time series of electron density at the altitude of $400 \mathrm{~km}$ from the northward-directed ESR dish and the EISCAT VHF radar.

had clear poleward components between 0700 UT and 0810 UT (see Figure 1f), there were no clear plasma flow channels associated with the patches seen by the ESR, indicating that these features had settled into the polar cap convection pattern, i.e., that they had moved well poleward of the patch formation region [Cowley and Lockwood, 1992].
[5] The VHF radar covered the latitude range $68^{\circ}-76^{\circ}$ MLAT equatorward of the ESR field of view. The black curve superimposed on Figures $1 \mathrm{~h}-1 \mathrm{k}$ represents the $\mathrm{Ti}=1600 \mathrm{~K}$ isocontour line, extracted from the equatorward edge of the high $\mathrm{Ti}$ band in Figure $1 \mathrm{j}$. This $\mathrm{Ti}=1600 \mathrm{~K}$ boundary is suggested to be the open-closed field line 


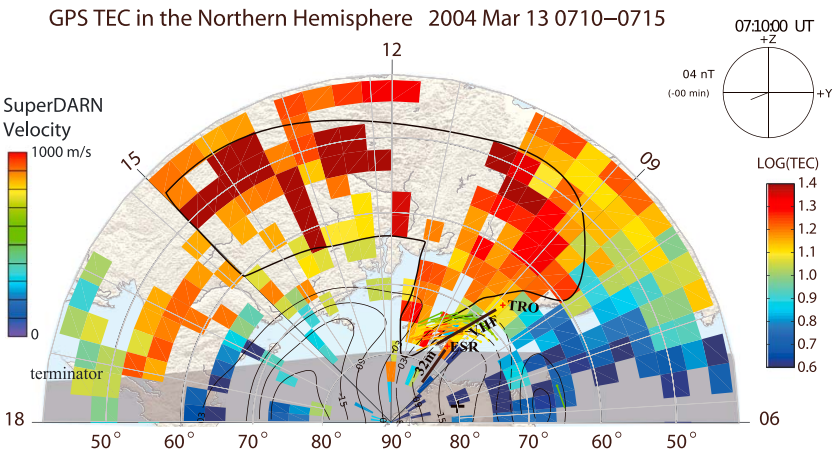

Figure 2. Combined GPS TEC and convection observations are projected on geomagnetic grids and a coast map for the dayside sector (magnetic latitude/ MLT coordinates; $10^{\circ}$ latitude circles; with noon at the top), with the daynight terminator at $100 \mathrm{~km}$ altitude. Five minute averaged 2-D map of the 10-based logarithmic vertical TEC observations binned by latitude and longitude for $0710-0715$ UT are displayed with the simultaneous, independent convection pattern and drift velocity vectors derived from the Northern Hemispheric SuperDARN observations. These velocities are fitted velocities from map-potential algorithm at the location of actual radar measurements, which can be up to $50 \%$ smaller for the fast flows than the raw radar data, or flows observed by DMSP [e.g., Xu et al., 2008], due to spatial filtering and smoothing in the spherical harmonic fitting process. The continuous black line outlines the boundaries of the enhanced density between which TEC generally exceeded 1.5 times higher or more than that in the background. The lines of sight of the poleward looking low-elevation beam $(32 \mathrm{~m}$ dish) of the ESR and of the beam of the EISCAT VHF radar are indicated by the solid black lines.

boundary (OCFLB) on the basis of previous modeling work which has shown that a band of high ion temperatures, enhanced through frictional heating, will form on the lowlatitude side of a contracting OCFLB and on the high-latitude side of an expanding boundary [Lockwood et al., 1988; Moen et al., 2004]. Note that the boundary is clearest in the ion temperature and ion flow data, both of which are expected to be essentially height independent and hence reliably reflect the latitudinal structure of the boundary and its motions. This contrasts with the electron density data, shown in Figure 1h, where the variation with range is almost certainly dominated by altitude dependence. The OCFLB, as defined from the ion temperature data, can be seen to have contracted progressively poleward, as the polar cap contracted due to the progressive northward turning in IMF $B_{Z}$ after about 0740 UT. Before about $0750 \mathrm{UT}$, the electron density showed clear PMPCEs (see Figure 1h) across the OCFLB, together with transitory enhancements in the electron temperature (see Figure 1i), both of which are highlighted by solid black lines and marked by the red numbers I-III. After about 0750 UT, the electron density appeared smooth and relatively high between about $70^{\circ}$ and $73^{\circ}$ magnetic latitude (at an altitude between about 200 and $300 \mathrm{~km}$ ), suggesting that the formation of PMPCEs in the EISCAT VHF viewing area ceased as the IMF began to turn northward.

[6] Figure 2 shows the combined GPS-based vertical total electron content (TEC) [Coster et al., 2003] and convection observations. We can see that there was a clear region of enhanced electron density at around $50^{\circ}$ magnetic latitude, which appeared to extend poleward, following the convection streamlines into polar latitudes in the morning sector, and entering the polar cap in the vicinity of Svalbard (missing pixels denote a lack of GPS TEC coverage in that region). This feature can readily be interpreted as a TOI. The ionospheric convection measurements from both the SuperDARN and EISCAT radars showed that there were clear eastward flow bursts across the line of sight of the VHF radar (equatorward of Svalbard) near to the poleward tip of the TOI. Poleward of this flow burst region, some "patch-like" electron densities are seen (best seen in the radar data presented above), suggesting that some mechanism, associated with the flow burst, had served to cut the TOI into patches.

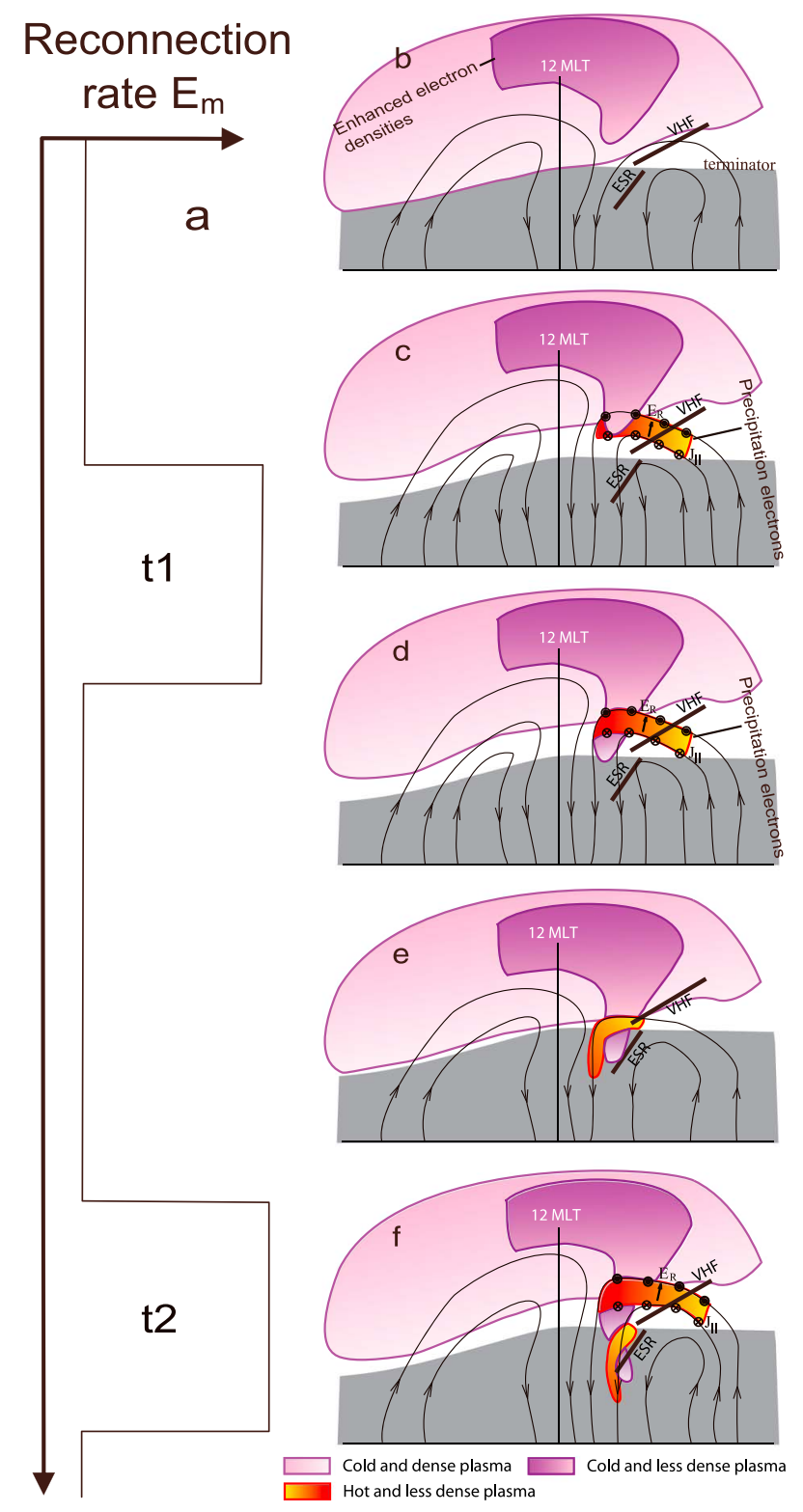

Figure 3. (a-f) Schematic showing how an elongated channel of high plasma density enters the cusp and polar cap region, forms a tongue of ionization (TOI), and is then segmented into patches by transient changes in flow channels generated by pulsed dayside magnetopause reconnection. 


\section{Discussion}

[7] We will first discuss the linkage between PMPCEs observed by the two EISCAT radars and then we will propose a possible patch formation mechanism to explain the observations. To avoid the altitude profile information folded into the radar electron density plots, Figure $1 \mathrm{~g}$ compares the electron densities observed at $400 \mathrm{~km}$ by the VHF (blue plus line) and the ESR (red star line) radars. In this panel, the VHF radar data have been shifted by $27 \mathrm{~min}$, which is the estimated time for features to propagate from the VHF to the ESR radar at the same altitude. It is interesting to note that the PMPCE electron densities observed by the EISCAT VHF are significantly lower than the PMPCE densities observed by the ESR (at higher latitude). In fact, the PMPCE electron densities measured by the VHF radar correspond to the density minima between the PMPCEs measured by ESR at the similar altitude (highlighted by the magenta circles in Figure 1g). There are four different plasma regimes: (1) background of cold and low density plasma at lower latitudes observed in the GPS TEC and EISCAT VHF; (2) enhanced electron densities, consisting of cold dense plasma in the GPS TEC data constituting the source of the TOI; (3) PMPCEs in the VHF radar data, possibly created by cusp reconnection/precipitation events, which then evolved poleward to form the minima between the polar cap patches measured by the ESR; and (4) the background plasma in the polar cap seen after 0825 UT by the ESR, characterized by very low temperatures and densities.

[8] As a possible explanation, we suggest that the $F$ region density structuring was the result of two interacting processes, shown schematically in Figure 3. Figure 3a shows the time history of the reconnection rate, while Figures $3 b-3 f$ show the time history of the event, with time increasing from the top to the bottom of the figure. The different plasma regimes described above are color coded. The lilac to white shading represents the underlying photoionized plasma at lower latitudes (regime 1 above), within which the purple to pink shading represents the colder, denser plasma which went on to form the TOI (regime 2). The yellow to red shading indicates the hotter plasma of the PMPCE events originating in the vicinity of the OCFLB (regime 3 ), while the grey shading represents the cold, low-density plasma of the polar cap (regime 4). The solid black lines represent the view fields and look directions of the EISCAT VHF and ESR radars.

[9] Regime 2 plasma is continuously brought toward the cusp inflow region is initially located at the OCFLB. When the first reconnection pulse occurs (at time t1), the OCFLB jumps equatorward to span across a region of high density plasma, which then becomes entrained into the high-latitude cross-polar convection, creating a TOI (Figure 3b). The reconnection event is, however, also characterized by flow bursts and precipitation, in such a way that regime 3 plasma, injected in the region of the OCFLB, becomes interspersed with the denser plasma of regime 2 enhanced by electron precipitation in the cusp region (Figure 3c). Moen et al. [2001] documented that plasma accumulation due to particle impact ionization of poleward moving cusp auroral forms to be of the order $10^{-11} \mathrm{~m}^{-3}$ as observed for regime 3 plasma here. That the electron temperatures in the PMPCE events observed by EISCAT VHF appeared to be enhanced while the electron temperatures were lower in the density minima between events (Figure 1i) are consistent with auroral electron precipitation in the cusp as the generation mechanism for the low-density PMPCEs. After the first reconnection pulse ceases, the flow burst decays away, by which time the high-density patch has become fully detached from the TOI, with the hotter, but less dense, plasma segmenting it into patches (Figure $3 \mathrm{~d}$ ). The OCFLB then relaxes back poleward, carrying both the patch and the region of precipitation into the polar cap (Figure 3e), and the high-density regime 2 plasma follows the OCFLB as well. In the same way, the creation of the second patch is associated with the second reconnection pulse (at $\mathrm{t} 2$ in Figure 3a), with the patch again being segmented from the TOI by the eastward flow burst associated with the second reconnection event.

\section{Conclusion}

[10] We have presented clear evidence for two types of PMPCEs: Type L (low density) resulting from particle precipitation and Type $\mathrm{H}$ (high density) resulting from segmentation of an enhanced tongue of ionization (TOI). In this case, the observed high-latitude density structure results from the interaction of these two types of density enhancement. We find our observations consistent with the proposal that the Type L PMPCEs observed by the EISCAT VHF radar were produced by particle precipitation related to reconnection events, and subsequently convected poleward along cusp flow channels. These Type L PMPCEs were of the same density as the minima between the Type H PMPCEs observed by the EISCAT Svalbard Radar. Our inference is that the Type L and Type H PMPCEs became interspersed as a result of Type L PMPCEs segmenting a region of high-density plasma, such as a TOI, thus forming Type H PMPCEs. This observation emphasizes the complex role that cusp flow channel events may play in structuring electron density at high latitudes. Furthermore, the case study presented here constitutes the first clear evidence that TOI patches may also enter the polar cap from the dawn convection cell, as earlier suggested by Moen et al. [2004, 2008]. All of these insights into the formation of high-latitude density structure provide key scientific knowledge for the development of physically based Global Navigation Satellite System scintillation forecasts for the polar cap.

[11] Acknowledgments. This work is supported by the National Basic Research Program of China (grant 2012CB825603), the National Natural Science Foundation of China (grants 41274149, 41104091, 41031064, and 40890164), and the International Collaboration Supporting Project, Chinese Arctic and Antarctic Administration (IC201112), and the project is supported by the Specialized Research Fund for State Key Laboratories, and Ocean Public Welfare Scientific Research Project, State Oceanic Administration People's Republic of China (201005017). J. Moen is supported by the Norwegian Research Council and COST ES803. M. Lester is supported by STFC grantST/H002480/1. We thank the MIT Haystack Observatory for generating GPS TEC data and making them available through the Madrigal Database (http://madrigal.haystack.mit.edu/) and the NASA CDAWeb site for supplying us the solar wind and IMF data from the ACE spacecraft.

[12] The Editor thanks two anonymous reviewers for their assistance in evaluating this paper.

\section{References}

Carlson, H. C. (2012), Sharpening our thinking about polar cap ionospheric patch morphology, research, and mitigation techniques, Radio Sci., 47, RS0L21, doi:10.1029/2011RS004946.

Coster, A. J., J. Foster, and P. Erickson (2003), Monitoring the ionosphere with GPS: Space weather, GPS World, 14(5), 42. 


\section{ZHANG ET AL.: PATCH SEGMENTATION OF TOI}

Cowley, S. W. H., and M. Lockwood (1992), Excitation and decay of solar wind-driven flows in the magnetosphere-ionosphere system, Ann. Geophys., 10, 103-115.

Crowley, G. (1996), Critical review of ionospheric patches and blobs, in Review of Radio Science 1992-1996, edited by W. R. Stone, pp. 619-648, Oxford Univ. Press, Oxford, U. K.

Foster, J. C., et al. (2005), Multiradar observations of the polar tongue of ionization, J. Geophys. Res., 110, A09S31, doi:10.1029/2004JA010928.

Knudsen, W. C. (1974), Magnetospheric convection and the high-latitude F2 ionosphere, J. Geophys. Res., 79(7), 1046-1055.

Liu, Z. X., C. P. Escoubet, Z. Pu, H. Laakso, J. K. Shi, C. Shen, and M. Hapgood (2005), The Double Star mission, Ann. Geophys., 23, 2707-2712.

Lockwood, M., and H. C. Carlson (1992), Production of polar cap electron density patches by transient magnetopause reconnection, Geophys. Res. Lett., 19(17), 1731-1734.

Lockwood, M., S. W. H. Cowley, H. Todd, D. M. Willis, and C. R. Clauer (1988), Ion flows and heating at a contracting polar cap boundary, Planet. Space Sci., 36, 1229-1253.

Lockwood, M., et al. (2005), Motion of the dayside polar cap boundary during substorm cycles: II. Generation of poleward-moving events and polar cap patches by pulses in the magnetopause reconnection rate, Ann Geophys., 23, 3513-3532.

MacDougall, J., and P. T. Jayachandran (2007), Polar patches: Auroral zone precipitation effects, J. Geophys. Res., 112, A05312, doi:10.1029/ 2006JA011930.

Moen, J., A. P. van Eyken, and H. C. Carlson (2001), EISCAT Svalbard Radar observations of ionospheric plasma dynamics in relation to dayside auroral transients, J. Geophys. Res., 106(A10), 21,453-21,461.

Moen, J., et al. (2004), The dynamics and relationships of precipitation, temperature and convection boundaries in the dayside auroral ionosphere, Ann. Geophys., 22, 1973-1987.

Moen, J., X. C. Qiu, H. C. Carlson, R. Fujii, and I. W. McCrea (2008), On the diurnal variability in F2-region plasma density above the EISCAT Svalbard radar, Ann. Geophys., 26, 2427-2433.

Weber, E. J., et al. (1984), F layer ionization patches in the polar cap, J. Geophys. Res., 89(A3), 1683-1694.

$\mathrm{Xu}$, L. et al. (2008), A 2-D comparison of ionospheric convection derived from SuperDARN and DMSP measurements, Adv. Space Res., 42, 1259-1266.

Zhang, Q. -H., et al. (2011), On the importance of interplanetary magnetic field $|\mathrm{By}|$ on polar cap patch formation, J. Geophys. Res., 116, A05308, doi:10.1029/2010ja016287. 